\title{
An Exploration of the Concepts of Loss and Grief as Stress Responses in Middle Eastern Parents of Children with Cancer
}

\author{
Rami Masa'Deh ${ }^{1}$, Carol Hall ${ }^{2} \&$ Jacqueline Collier ${ }^{3}$ \\ ${ }^{1}$ School of Nursing, Applied Science Private University, Amman, Jordan \\ ${ }^{2}$ School of Health Sciences, The University of Nottingham, Queens Medical Centre, Nottingham UK \\ ${ }^{3}$ School of Psychology, University of East Anglia, Queen's Building, Norwich Research Park, Norwich, UK \\ Correspondence: Rami Masa'Deh, Associate Professor, School of Nursing, Applied Science Private University, \\ Amman 11934, Jordan. Tel: 962-79-6067657. E-mail: r_masadeh@asu.edu.jo
}

Received: August 15, 2016

Accepted: December 5, 2016 Online Published: March 13, 2017

doi:10.5539/gjhs.v9n6p97

URL: https://doi.org/10.5539/gjhs.v9n6p97

\begin{abstract}
Background: Caring for a child with cancer has been found to have profound and sometimes long-lasting negative psychological effects on parents. Grief of those parents was less examined in Jordan than America and Europe. Many parents in studies carried out in other countries experienced shocke, disbelief and guilt about their child's diagnosis. This pattern is similar to the stages of grief as identified by Kubler-Ross model and this association has been identified as potentially benefitting from being further explored in differing cultures such as the experiences of Middle-Eastern parents. Therefore, this study aims to examine the experience of mothers and fathers of children diagnosed with cancer in Jordan and explore whether their narratives reflect grieving as understood through the theoretical constructs of Kubler-Ross. This study investigated the applicability of the Western grief model in an Arab community.
\end{abstract}

Methods: Using a qualitative approach, 24 parents of children with cancer were interviewed. The participants were recruited recruited from the biggest cancer specialist centre in Jordan. Thematic analysis was use to analyse their results.

Results: It was clear that the emotional reactions of Jordanian parents of children with cancer fit with Kubler-Ross' loss and grief model. Jordanian parents of children with cancer reported experiencing denial, anger, depression, bargaining and acceptance.

Conclusions: The results of this study provide evidence that the reactions of Jordanian parents offer a clear direction for the applicability of care strategies from other communities with culturally different backgrounds. Jordanian parents, health care providers and particularly nurses should be aware that parental grieving process as identified by Kubler-Ross' model can be experienced by those receiving a diagnosis of a life-threatening illness in their child and is individualised, normal and most often ends with acceptance. Therefore, a supporting framework to those parents should take into consideration their stage of grief. Nurses and parents should know that they do not have to push the stages; acceptance will be reached once the individual is ready

Keywords: cancer, child, grief, Jordan, parent

\section{Introduction}

Caring for a child with cancer has been found to have profound and sometimes long-lasting negative effects on parents (Boman, Lindahl, \& Bjork, 2003; Lindahl Norberg \& Boman, 2008; Masa'Deh, Collier, Hall, \& Alhalaiqa, 2013). Research predominantly carried out in Europe and North America illustrates that parents of children with cancer diagnoses must deal with a complex set of physical, emotional and psychological challenges alongside practical and financial issues (Gravestock, McDowell, \& Vale, 2011; Long \& Marsland, 2011; Rodriguez et al., 2012; Warner et al., 2011). Subsequent work has discovered that these issues are also found within the experiences of families of children with cancer in Jordan (Ekhlas Al-Gamal \& Long, 2010; Masa'Deh et al., 2013; Saifan, Masa'Deh, Hall, \& Collier, 2014). Nonetheless, the means to manage or cope with stress is highly dependent upon the context in which it occurs and is perceived. Lazarus and Folkman (1984) suggest that 'stress' may result from an imbalance between an individual 'stressor' and the resources to buffer it. This is further recognised as being both 'externally' focussed (responding to extrinsic events) and internally focused, whereby coping includes the 
need to respond effectively to an 'emotional' response. The way in which individuals conceptualise their stress and use resources to manage this is thus deeply contextually situated and may be different across cultures.

While a substantial body of literature exists on parental responses to living with a child with cancer in Western contexts, there has been less research on these phenomena in Arab-Islamic cultures. There is limited evidence about whether the experiences of those parenting a seriously ill child in the Middle-East and North Africa (MENA) can be considered using theoretical 'coping' constructs identified in research with Western parents. This is important when considering the outcome of 'stress' on families in MENA and for nurses who must maximise the potential for families to establish effective means for adapting and coping with 'stressors'.

This paper builds upon previous work undertaken by the authors to explore stress-related perceptions of parents with children with cancer in Jordan. In this paper, the exploration of data compares well established and critically appraised conceptual models to illuminate and explore their relevance to Arab-Islamic life in light of the findings of one mixed methods study. Chosen models include Folkman and Lazarus (1984) as the underpinning theoretical framework relating to stress and stress management, and in light of the findings of the study of parents of children with cancer in Jordan, adds in further insights emanating from a review of the older grief and loss model by Kubler-Ross (1969).

\section{Background}

As part of a larger mixed-methods study exploring the psychological wellbeing of parents of Jordanian children with cancer, we explored stressors experienced by parents. This exploration was situated originally within the model of stress and coping devised by Folkman and Lazarus (1984), which is a robust model that meets the original aims of the work (to understand stress among parents of children with cancer). The model's focus upon internal and external elements enables exploration of both objective and subjective data. The model is universally critiqued and applied and also includes previous translation and application within a Jordanian context.

The study used a large quantitative survey using the Arabic version of the Perceived Stress Scale (PSS10) to assess parental perceived stress. This allowed parents to opt into a smaller qualitative interview study which employed a descriptive exploratory approach to gain more in-depth information to explain the perceived stress levels reported in the quantitative survey. Parts of the study were more fully reported (Masa'Deh et al., 2013; Saifan et al., 2014). A review of themes which emerged from the qualitative study led the researchers to re-evaluate the fit of the underpinning conceptual model used solely to explain the perceptions of parents of children with cancer in Jordan. In addition to Lazarus and Folkman (1984), it was found that other models which focussed upon concepts of loss and grief was helpful in illuminating new future directions.

During the interviews, parental responses included considerable elements of emotional content. The emotional content of the narratives of these Arab-Islamic parents particularly appeared to focus upon concepts of grief and loss. This paper sets out to explore these narratives in the context of established Western contexts and make suggestions about the applications of such theories and their predictive outcomes for Middle Eastern care giving. Our research team's observation of expressed grief following an established theoretical construct is potentially important, as there has been limited research among parents of children with life-threatening illness in Jordan or other countries throughout MENA.

\section{Review of Literature}

A literature search revealed only four papers within a series of studies addressing anticipatory grief among parents of Jordanian children with either cerebral palsy or cancer: (Al- Gamal \& T., 2013; E. Al-Gamal, 2013; Ekhlas Al-Gamal, Long, \& Livesley, 2009; Al - Gamal \& Long, 2010). Al-Gamal's papers developed, tested and then used modified versions of the Marwit and Meuser Caregiver Inventory to assess parental levels of anticipatory grieving. Meuser \& Marwit (2001) originally introduced their model of grief specifically for those caring for someone with Alzheimer's disease and developed their anticipatory grief measure which was made up of three subscales: personal sacrifice and burden; heartfelt sadness and longing; and worry and felt isolation. Al-Gamal and Livesley (2009) compared the grief of 70 Jordanian parents ( 57 mothers and 13 fathers) of a child newly diagnosed with cancer (i.e. less than two months) with 70 parents (42 mothers and 28 fathers) whose child was diagnosed 6-12 months earlier. The study found that $98 \%$ of those parenting newly diagnosed children agreed that they wished that they were dreaming. Approximately $70 \%$ of them agreed that they did not accept the cancer diagnosis. The majority reported anger, isolation and sadness. However, there was an effect with time, and those who were parenting a child diagnosed between 6 and 12 months were found to have less intensity of grief. The theoretical constructs of the instrument used by the Al-Gamal studies differed from those of Kubler-Ross, but the findings support each other. 
The Kubler-Ross model is a robust grief and loss model that describes the grieving process in five stages or responses (Davies, 2004). The stages are applied to people suffering from a major loss such as the death of a loved one, divorce, the onset of a disease or chronic illness (loss of health), and includes loss of expectations, as well as many tragedies and disasters (e.g. parenting a child with cancer). The five stages are recognised as denial, anger, bargaining, depression and finally acceptance. Kubler-Ross believed that these steps may not necessarily come in the above order and that there is a possibility that affected individuals may not experience all five stages. A review of 34 studies conducted by Smith et al. (2013) focused on parental experiences when having a child diagnosed with a long-term medical condition found that parents experienced several emotions such as shock, disbelief and anxiety of having a child diagnosed with a chronic illness. These grief reactions often disappeared if parents accepted the reality otherwise parents may refuse to believe their child's situation. Therefore, parents expressed various emotional reactions such as anger, blame and/or guilt (Smith et al., 2013). The grief and loss paradigm assumed that parents undergo (i.e. go through the experiences of) the predictable stages of grief and ultimately reach the last stage of acceptance; however, a failure to do so would manifest a serious dysfunction, with profound health consequences. Thus, professional psychological involvement with the family, such as a grief counsellor, is recommended to assist parents to work through their grief (Davies, 2004).

We could find no qualitative papers to further deepen understanding of the parental experiences in Jordan. Many parents in studies carried out in other countries felt shock, disbelief and guilt about their child's diagnosis. This pattern is similar to the stages of grief as identified by Kubler-Ross (2009) and this relationship has been identified as something to be explored with reference to different cultural contexts (Davies, 2004), such as the experiences of Middle-Eastern parents. Therefore, this study aims to examine the experience of mothers and fathers of children diagnosed with cancer in Jordan and explore whether their narratives reflect grieving as understood through the theoretical constructs of Kubler-Ross.

\section{Methodology}

\subsection{Setting the Context}

Participants were recruited as part of a larger mixed-methods study in Jordan (Masa'Deh et al., 2013). Those parents in this qualitative part of the research were all recruited from a single hospital, the biggest cancer specialist centre in Jordan, treating more than $90 \%$ of the overall childhood cancer cases (Jordan National Cancer Registry, 2011).

\subsection{Theoretical Framework}

The theoretical paradigm underpinning this overall research project was one of pragmatism (Maxcy, 2003). While previous papers reported elsewhere focussed upon quantitative investigation (Masa'Deh et al., 2013; Saifan et al., 2014), this paper reports upon the qualitative aspect which collected data through semi-structured interviews. The aim of these interviews was to explore grief using in-depth and illustrative instruments to explore 12 couples' perceptions of the stressors involved in caring for a child with cancer in Jordan, with inclusion of their interactions and perceptions of the physical world as well as the social world, which might include language, culture, human institutions and thoughts. The narratives of the parents interviewed were analysed using thematic analysis, and initial reading of the transcripts led to identification of themes which included aspects of the grief and loss model as developed by Kubler-Ross (1969). The interviews were undertaken with parents individually and their responses were compared as couples. This enabled both the individual's perspective and a further consideration of the joint nature of the experience of caring for their child.

\subsection{Ethical Considerations}

This study was a part of a larger study that investigated parental experiences when having a child with cancer. The study was approved by the research ethics committee at the cancer centre concerned and permission to proceed with the research was gained.

The researcher informed participants both verbally and in writing about their right to withdraw from the study at any time without giving a reason, causing no penalty or loss of benefits to them or their ill children. Data gained from the study was kept in an approved secure place of storage only accessible by the researchers. The researcher gave the participants full information about the purpose of the data they contributed and advised them that the data will be destroyed seven years after the completion of the study.

\subsection{Data Collection Procedure}

An interview reply slip was attached with the participant information package (invitation letter, information sheet and the consent form) whereby each participant could respond, demonstrating their desire to be considered in the 
study. Before conducting the interview, the researcher secured written consent for the study, which included consent for using quotes and consent for publishing their quotes.

The researcher contacted couples who volunteered to be interviewed and arranged with them for a convenient time and place for each of their interviews. The researcher (interviewer) was trained to conduct this kind of interview using an approved semi-structured schedule. With the permission of the participants, all interviews were undertaken in a special room prepared for research purposes and were audio-recorded. The interviews were conducted in Arabic (the official language in Jordan). Each interview took between 50 to 70 minutes, depending on the participants' story and their responses. Only the participant and the researcher were present at the interview time (no participants asked to be accompanied by someone during the interview, although this opportunity was offered to them). Additionally, a mother and a father forming a couple did not interact with each other between the interviews, as the researcher interviewed them immediately after each other. These measures were undertaken to ensure the independency of each parent's response.

At the beginning of each interview, the interviewer introduced himself and then started by asking the participants a general question regarding their experiences of having a child with cancer. All interviews began with a general question, for example; 'Could you please tell me about your experience of having a child with cancer?' This encouraged participants to talk about their experiences and provided background information about their present condition. Interviews were guided by an interview schedule. The researcher showed an interest in their stories and views, and confirmed that what has been said was completely confidential and their responses would be coded and maintained anonymously, in a way whereby couples could be linked together.

\subsection{Participants and Sampling}

Mothers and fathers parenting a child diagnosed with cancer in Jordan were approached. The inclusion criteria were:

- Parenting a child diagnosed with any type of cancer;

- Attending for his/her treatment or follow-up in the selected cancer centre; and

- Are able to speak, understand, read and write Arabic language.

The exclusion criteria were:

- Parents of dying children of those with a life expectancy of less than eight weeks.

All couples in the study were married and none were cohabiting, consistent with social norms of Jordan, where less than $1 \%$ of couples cohabit (WorldFamilyMap, 2014). A convenience sample of all families meeting the larger mixed-methods study criteria who attended the selected cancer centre during the data collection period (four months) were approached. Of the 305 couples who consented to take part in the overall study, a total of 42 mothers and 35 fathers agreed to proceed with the interview stage of the research. However, when arranged in couples, those mothers and fathers created a total of 19 couples. After that, 7 couples withdrew ( 3 couples did not answer their phones, 2 couples did not attend at the allocated interview time twice and 2 fathers were out of Jordan). As a result, the study involved 24 interviews with 12 couples parenting children with cancer in Jordan. The aim of including couples was to explore any differences between mothers and fathers in a family setting. The following table presents the profile of the participants. 
Table 1. Respondents' profile

\begin{tabular}{llllllll}
\hline ID & $\begin{array}{l}\text { Parents age } \\
\text { (mother/father) }\end{array}$ & $\begin{array}{l}\text { No. of } \\
\text { children }\end{array}$ & $\begin{array}{l}\text { Inpatient } \\
\text { or home }\end{array}$ & $\begin{array}{l}\text { Time since diagnosis } \\
\text { (months) }\end{array}$ & $\begin{array}{l}\text { Child's sex \& age } \\
\text { (years) }\end{array}$ & Cancer type \\
\hline 1 & $38 / 40$ & 6 & Home & 9 & F $(9.17)$ & Leukaemia \\
2 & $37 / 48$ & 5 & Home & 28 & M $(14.00)$ & Brain tumour \\
3 & $31 / 32$ & 2 & Home & 33 & M $(4.50)$ & Leukaemia \\
4 & $45 / 55$ & 4 & Inpatient & 13 & M $(2.00)$ & Leukaemia \\
5 & $35 / 44$ & 4 & Inpatient & 24 & M $(9.00)$ & Leukaemia \\
6 & $25 / 28$ & 1 & Home & 4 & M $(1.25)$ & Testis \\
7 & $41 / 49$ & 6 & Home & 9 & F $(1.25)$ & Eye \\
8 & $25 / 31$ & 1 & Home & 5 & M $(0.83)$ & Eye \\
9 & $36 / 37$ & 4 & Home & 4 & M $(0.67)$ & Others \\
10 & $39 / 42$ & 4 & Home & 152 & F $(13.00)$ & Others \\
11 & $45 / 53$ & 12 & Home & 44 & M $(10.50)$ & Leukaemia \\
12 & $20 / 34$ & 2 & Home & 8 & M $(2.83)$ & Leukaemia \\
\hline
\end{tabular}

\subsection{Data Analysis Process}

Data gained from the interviews was analysed manually by the researcher. Thematic analysis as identified by Boyatzis (1998) was used to analyse the data. The process of thematic analysis as applied in this study is briefly explained in the following steps:

- Transcription: transcription was done by the researcher immediately after the end of the interview. The researcher did not return the transcript to the interviewees to check because it was not feasible at the time.

- Double coding: this step was carried out by the researcher and another coder independently. They discussed differences in the codes and coding structure until agreement was reached.

- Translation: translation and back-translation by two bilingual health professionals whose native language is Arabic was undertaken. The researcher sat with the two professional linguists and discussed the translation until agreement on any ambiguous or problematic terms was reached.

- Data display: the researcher clarified themes in relation to the coded extracts, refined the specifics of each theme and gave clear definitions and names for each theme. Related quotes were presented under each theme.

The themes related to the stressors of caring for a child with cancer (i.e. treatment; new roles and responsibilities; isolation and self neglect; siblings and general family issues; financial issues) have been reported previously (Saifan et al., 2014). Emotional responses to the situation were found within the narrative and broadly fit with the five stages of the Kubler-Ross theory of grief, and (as she noted) the stages were not always linear.

\section{Findings and Interpretations}

\subsection{Denial}

The time of diagnosis was found to be very stressful for all interviewed mothers and fathers, and mothers were particularly forthcoming about this. Some parents responded to the event with extreme emotional intensity, whereas others had less severe emotional responses. Shock was the first emotion reported by the participants when the diagnosis was confirmed. Mothers and fathers admitted that they were shocked at the time of diagnosis, and some mothers lost consciousness upon hearing the cancer diagnosis, whereas others ran, shouted and cried. The next two quotes demonstrate how a mother and a father described this period of time:

... When we heard the news we both got shocked. My husband cried and I cried so much (my husband had more control on himself compared to me). I felt that the child would die. I immediately linked cancer to death. I did not know that there is a room for recovery and I have not heard before that there are people who were cured from cancer. (Mother3)

...The diagnosis was a shock for both of us. My wife cried and could not take it. It is very hard to hear such bad 
news. I cannot explain of how I felt at that time. It was a disaster. He is the first child for us and he is an angel. (Father6)

Other participants described the time of receiving the diagnosis like a dream from which they wanted to escape but they could not. They felt overwhelming despair, denied the diagnosis and feared their child's death. For instance, one mother said:

...All of this was like a dream...I did not believe it in the beginning...I did not want my son to go to the cancer hospital, but he had another spike of temperature so we went there and entered the hospital. This made me aware that it is not a dream, rather it is a horrible fact. (Mother3)

These findings are similar to those of Yeh (2003) who found that Taiwanese parents sometimes denied their child's diagnosis and searched for alternative explanations of illness from spiritual and religious beliefs. Despite numerous cultural factors that come into play in such scenarios (e.g. particular religious/spiritual expressions), there are similar parental reactions to hearing the cancer diagnosis of their children in various communities (Maurice-Stam, Oort, Last, \& Grootenhuis, 2008; Nicholas et al., 2009; Quintana, Wottrich, Camargo, \& Cherer, 2013).

\subsection{Anger}

Some interviewed parents blamed themselves for the cancer diagnosis of their child. Parents sought for internal explanations of the cancer diagnosis of their child and some started to devalue themselves. They thought that their child's cancer was their own fault and was caused by negligence in caring or unhealthy lifestyles, such as smoking or unhealthy eating habits. Therefore, it is important to provide parents with information about the disease immediately at the time of the diagnosis to help them understand the possible causes of the disease, and that having a child with cancer is not their fault. In this study, a few mothers and fathers had existential thoughts about why and how the child was diagnosed with cancer, as in the following examples:

...I was wondering of why me among all people?... And why my son among all children in the world? Why did this happen to us?... What did I do wrong in my life? And so on. (Mother5)

... When I heard the diagnosis of my child I became very angry and did not want to hear anything else. I did not realize that I left kicking the door behind me. (Father6)

Some interviewed mothers explained that they want their children to be happy, to recover from the disease and to grow up with good habits and behaviours. This is common in parents whose children are very ill as a means to solving their own feelings of guilt. This concern was raised by mothers more than fathers. The next quote reflects this issue:

...I was in a conflict between keep the ill child happy and to rear him, I want him to be good when he grows up. I do not want him to recover from the disease and acquire bad habits or attitude. Sometimes I had to be serious with him, scream, make discipline and act like any normal relation between mother and son but when I do so I blame myself for what I did. It is hard to do it, the fact that he has cancer and rearing him is a bit complicated and that really made me tired sometimes. (Mother5)

The findings indicate that parents may experience inward or outward reactions of anger when they first learn their child's diagnosis, consistent with Al-Gamal and Long (2010), who found parental self-blame was a theme that emerged in their data. Additionally, Smith et al. (2015) reviewed studies related to parental experiences when having a child with cancer and reported that anger was the most common emotional reaction of the parents in many reviewed studies.

\subsection{Bargaining}

In the current study, many interviewed parents bargained the health of their child. Some interviewed parents expressed that they would do anything in order to escape from their child being diagnosed with cancer. Some of them wished they could pay their money and have the health of their children in return, while many mothers and fathers said that they would prefer to have cancer themselves to save their children:

...Make this not happen, and in return I will pay all the money I have. (Father4)

...I wish if I am the one who was diagnosed with cancer but not my child. He is very young to be diagnosed with such a disease. (Mother7)

Although all parents in the current study admitted that they do not believe in alternative medicine as a cure, some of them used several kinds of herbs and/or a particular type of acupuncture to treat their ill child. The idea which was clearly stated by parents behind the use of such alternative therapy was that even if this alternative medicine 
did not have any benefit, it would not cause harm. This may reflect the bargaining stage of grief. For example, one father commented on this:

...I did my best and knocked on all doors looking for a cure to my son. I tried some herbs. If this herbal treatment does not work, I guess it will not harm him. It is only herbs, not chemotherapy!... I am sure that any alternative medicine will not be as harmful as the medical treatment. (Father3)

Bargaining was found to be a common behaviour in parents of children with serious disease and those undergoing painful medical procedures (Bluebond-Langner, Belasco, Goldman, \& Belasco, 2007; Cline et al., 2006; McCarthy \& Kleiber, 2006).

\subsection{Depression}

Having a child with cancer was found to be time-consuming for both parents, which affected not only the parental social relationships but also might affect their own personal needs. For example, a few interviewed mothers (but not fathers) admitted that they experienced a negative impact on their physical health and a lack of self-caring (characterised by weight changes), as they were stressed and had no time for themselves. In a world in which the role of mothers is constructed as being selfless, it was clearly difficult for mothers to give attention to their own needs. Some of them admitted that they were no longer taking care of their appearance as they had previously, because they did not have the time and/or the emotional ability to do so. Mothers stated that they spent the majority of their time with the ill child, either in hospital or at home and therefore having no time for their basic needs. Other participants highlighted the issue that having a child with cancer deprived them of their social environment and left them uninterested in caring for themselves. This may reflect the depression stage in grief. The following quotes explain this issue:

... It has been ages since I looked at myself in the mirror. I do not care of myself like before, such as my hair and nails. I spend most of my time with my child, why should I care about my appearance?! I do not care about myself as much as my child's health. (Mother4)

...I am too sad to do anything. I don't want to socialize with people; I don't even answer my phone calls. People will not cure my child. (Mother7)

Findings in the current study are similar to those of Cernvall et al. (2015), who investigated depression among 79 parents of children with cancer and reported that depression symptoms were very prevalent. Additionally, parental depression when having a child with cancer was also reported in a study in China (Chen et al., 2014).

\section{Acceptance}

In the current study, religious beliefs seemed to have helped the Jordanian parents to accept the diagnosis of their child. For example, many parents stated that everything is from God, and therefore they had to accept it without question, highlighting a religious belief common among Muslims. For instance, one interviewed father commented said:

...We are Muslims, as you know Islam says that God puts us in a hard situation to test us. I believe that everything is from God. Disease and cure are from God. Therefore, we must accept the disease, as we can do nothing to prevent it, and we should look for the best treatment. (Father2)

The parents' views in this study highlighted how a previous experience of dealing with cancer may affect how they think and react when hearing the cancer diagnosis of their child. Previous experiences of cancer with a close relative (such as a mother or a father) or of death in general were variously found to be helpful or unhelpful for different participants. Whilst one interviewed mother found it helpful that she had some knowledge about the disease and how to act with it, other parents stated that the previous experience affected them negatively, as they imagined everything that had happened in the past with their relatives (such as suffering, pain, death and the whole scenario) could happen for their ill child and they would have to relive that experience. Although parents knew that childhood cancer is quite different from other types of cancer, they thought that suffering and death are the common and inevitable results of all types of cancer. For instance, one mother raised this issue:

...I know what people go through when they are diagnosed with cancer. My uncle suffered and died from cancer. I know this type is different but I am really worried that my son would pass in a similar experience of suffering, pain and death... my uncle had to do a lot of aggressive medical tests every now and then. He used to spend most of his time in the hospital. (Mother6)

The findings of this study show that the time of announcing the diagnosis was considered a very critical and stressful for the parents. However, such stress may decrease over time, as noted by the following two parents. It is important to note that the first quote reflects a father had a daughter who was diagnosed with cancer ten years' 
previously. This couple had the longest period since the child was diagnosed with cancer among all interviewed parents. This may indicate that stress may decrease over time and/or in form of survivorship and/or reaching the acceptance stage of grief:

...It is all right. Although it was very hard to believe in the diagnosis in the beginning but I think that time can heal everything. You get over the hard situations and you get used to everything by time. (Father10)

...Having a child with cancer has become part of our life. We hope that things will change one day but we are aware that everything may happen to our child in the future. (Mother11)

Previous researchers such as Burke et al. (2014) and Polat et al. (2015) showed that many mothers and fathers accepted the cancer diagnosis of their children and also accepted the side effects and the consequences of the treatment. The time needed for the reaching the acceptance stage was found to be individualised, and varied among different parents (Polat et al., 2015).

\section{Conclusion}

This study investigated the applicability of the Western grief model in an Arab community. It was clear that the emotional reactions of Jordanian parents of children with cancer fit with the loss and grief model of Kubler-Ross. This work explored a previously unaddressed area and uncovered many insights into the experiences of Arab-Islamic parents and the way in which they experience grief when informed that their child has cancer. Currently, this new work provides evidence that we do not have to make that assumption. The reactions of these parents offer a clear direction for the applicability of care strategies from other communities with culturally different backgrounds.

Jordanian parents, health care providers and particularly nurses should be aware that the parental grieving process, as identified by the Kubler-Ross model, is individualised, normal and most often ends with acceptance. Therefore, a supporting framework to those parents should take into consideration their stage of grief. Nurse and parents should know that they do not have to push the stages, and acceptance will generally be reached once the individual is ready.

\section{Competing Interests Statement}

The authors declare that there is no conflict of interests regarding the publication of this paper.

\section{References}

Al- Gamal, E., \& T., L. (2013). The MM-CGI Cerebral Palsy: modification and pre-testing of an instrument to measure anticipatory grief in parents whose child has cerebral palsy. Journal of Clinical Nursing.

Al-Gamal, E. (2013). Quality of life and anticipatory grieving among parents living with a child with cerebral palsy. International Journal of Nursing Practice, 19(3), 288-294. https://doi.org/10.1111/ijn.12075

Al-Gamal, E., \& Long, T. (2010). Anticipatory grieving among parents living with a child with cancer. Journal of Advanced Nursing, 66(9), 1980-1990. https://doi.org/10.1111/j.1365-2648.2010.05381.x

Al-Gamal, E., Long, T., \& Livesley, J. (2009). Development of a modified instrument to measure anticipatory grieving in Jordanian parents of children diagnosed with cancer: The Marwit and Meuser caregiver inventory childhood cancer. Cancer nursing, 32(3), 211-219. https://doi.org/10.1097/NCC.0b013e31819a2ae4

Al - Gamal, E., \& Long, T. (2010). Anticipatory grieving among parents living with a child with cancer. Journal of Advanced Nursing, 66(9), 1980-1990. https://doi.org/10.1111/j.1365-2648.2010.05381.x

Bluebond-Langner, M., Belasco, J. B., Goldman, A., \& Belasco, C. (2007). Understanding Parents' Approaches to Care and Treatment of Children with Cancer When Standard Therapy Has Failed. Journal of Clinical Oncology, 25(17), 2414-2419. https://doi.org/10.1200/JCO.2006.08.7759

Boman, K., Lindahl, A., \& Bjork, O. (2003). Disease-related Distress in Parents of Children with Cancer at Various Stages after the Time of Diagnosis. Acta Oncologica, 42, 137-146. https://doi.org/10.1080/02841860310004995

Boyatzis, R. E. (1998). Transforming Qualitative Information "Thematic Analysis and Code Development". SAGE.

Burke, K., Muscara, F., McCarthy, M., Dimovski, A., Hearps, S., Anderson, V. et al. (2014). Adapting acceptance and commitment therapy for parents of children with life-threatening illness: Pilot study. [doi:10.1037/fsh0000012]. Families, Systems, \& Health, 32(1), 122-127. https://doi.org/10.1037/fsh0000012

Cernvall, M., Skogseid, E., Carlbring, P., Ljungman, L., Ljungman, G., \& von Essen, L. (2015). Experiential 
Avoidance and Rumination in Parents of Children on Cancer Treatment: Relationships with Posttraumatic Stress Symptoms and Symptoms of Depression. Journal of Clinical Psychology in Medical Settings, 1-10.

Chen, J., Liu, Y., Cai, Q., Liu, Y., Wang, T., Wang, J. et al. (2014). Depression in parents of children with leukemia in southern China accompanied by the prevalence of type D personality. Supportive Care in Cancer, 22(5), 1277-1286. https://doi.org/10.1007/s00520-013-2082-2

Cline, R. J. W., Harper, F. W. K., Penner, L. A., Peterson, A. M., Taub, J. W., \& Albrecht, T. L. (2006). Parent communication and child pain and distress during painful pediatric cancer treatments. Social Science \& Medicine, 63(4), 883-898. https://doi.org/10.1016/j.socscimed.2006.03.007

Davies, R. (2004). New understandings of parental grief: literature review. Journal of Advanced Nursing, 46(5), 506-513. https://doi.org/10.1111/j.1365-2648.2004.03024.x

Folkman, S. (1984). Personal Control and Stress and Coping Processes: A Theoretical Analysis. Journal of Personality and Social Psychology, 46(4), 839-852. https://doi.org/10.1037/0022-3514.46.4.839

Gravestock, H., McDowell, K., \& Vale, D. (2011). Counting the Costs of Cancer. United Kingdom: CLIC Sargent. Jordan National Cancer Registry. (2011). Annual Report of Ministry of Health in Jordan. Amman.

Kübler-Ross, E. (1969). On death and dying. New York: Macmillan.

Kübler-Ross, E. (2009). On death and dying: What the dying have to teach doctors, nurses, clergy and their own families. Taylor \& Francis.

Lazarus, R. S., \& Folkman, S. (1984). Stress, appraisal, and coping / Richard S. Lazarus, Susan Folkman. New York: Springer Pub. Co.

Lindahl Norberg, A., \& Boman, K. K. (2008). Parent distress in childhood cancer: A comparative evaluation of posttraumatic stress symptoms, depression and anxiety. Acta Oncologica, 47(2), 267-274. https://doi.org/10.1080/02841860701558773

Long, K. A., \& Marsland, A. L. (2011). Family adjustment to childhood cancer: A systematic review. Clinical Child and Family Psychology Review, 14(1), 57-88. https://doi.org/10.1007/s10567-010-0082-z

Masa'Deh, R., Collier, J., Hall, C., \& Alhalaiqa, F. (2013). Predictors of Stress of Parents of a Child with Cancer: A Jordanian Perspective. Global Journal of Health Science, 5(6), 81. https://doi.org/10.5539/gjhs.v5n6p81

Maurice-Stam, H., Oort, F., Last, B., \& Grootenhuis, M. (2008). Emotional Functioning of Parents of Children with Cancer: The First Five Years of Continuous Remission After the End of Treatment. Psycho-Oncology, 17(5), 448-459. https://doi.org/10.1002/pon.1260

Maxcy, S. J. (2003). 'Paragmatic Threads in Mixed Methods Research in the Social Sciences: The Search of Multiple Modes of Inquiry and the End of the Philosophy of Formalism'. In A. Tashakkori \& C. Teddlie (Eds.), Handbook of Mixed Methods in Social \& Behavioral Research (pp. 51-91). California: Thousand Oaks: Sage.

McCarthy, A. M., \& Kleiber, C. (2006). A Conceptual Model of Factors Influencing Children's Responses to a Painful Procedure When Parents are Distraction Coaches. Journal of Pediatric Nursing, 21(2), 88-98. https://doi.org/10.1016/j.pedn.2005.06.007

Meuser, T. M., \& Marwit, S. J. (2001). A Comprehensive, Stage-Sensitive Model of Grief in Dementia Caregiving. The Gerontologist, 41(5), 658-670. https://doi.org/10.1093/geront/41.5.658

Nicholas, D. B., Gearing, R. E., McNeill, T., Fung, K., Lucchetta, S., \& Selkirk, E. K. (2009). Experiences and resistance strategies utilized by fathers of children with cancer. Social Work in Health Care, 48(3), 260-275. https://doi.org/10.1080/00981380802591734

Polat, S., Güdücü Tüfekci, F., Küçükoğlu, S., \& Kobya Bulut, H. (2015). Acceptance-rejection levels of the Turkish mothers toward their children with cancer. Collegian.

Quintana, A. M., Wottrich, S. H., Camargo, V. P., \& Cherer, E. d. Q. (2013). Childhood Cancer: Meanings Attributed to the Disease by Parent Caregivers. Paidéia (Ribeirão Preto), 23, 253-261. https://doi.org/10.1590/1982-43272355201313

Rodriguez, E., Dunn, M., Zuckerman, T., Vannatta, K., Gerhardt, C., \& Compas, B. (2012). Cancer-related sources of stress for children with cancer and their parents. Journal of Pediatric Psychology., 37(2), 185-197. https://doi.org/10.1093/jpepsy/jsr054

Saifan, A., Masa'Deh, R., Hall, C., \& Collier, J. (2014). Experiences of Jordanian Mothers and Fathers of Children 
with Cancer. Journal of American Science, 10(8), 29-39.

Smith, J., Cheater, F., \& Bekker, H. (2013). Parents' experiences of living with a child with a long-term condition: a rapid structured review of the literature. Health Expectations, 18(4), 452-474. https://doi.org/10.1111/hex.12040

Smith, J., Cheater, F., \& Bekker, H. (2015). Parents' experiences of living with a child with a long-term condition: a rapid structured review of the literature. Health Expectations, 18(4), 452-474. https://doi.org/10.1111/hex.12040

Warner, C. M., Ludwig, K., Sweeney, C., Spillane, C., Hogan, L., Ryan, J. et al. (2011). Treating Persistent Distress and Anxiety in Parents of Children With Cancer: An Initial Feasibility Trial. Journal of Pediatric Oncology Nursing, 28(4), 224-230. https://doi.org/10.1177/1043454211408105

WorldFamilyMap. (2014). Mapping family change and child well-being outcomes. Retrieved 14 september, 2015, from http://worldfamilymap.org/2014/wp-content/uploads/2014/06/WFM-2014-Final_ForWeb.pdf

Yeh, C.-H. (2003). Dynamic coping behaviors and process of parental response to child's cancer. Applied nursing research: ANR, 16(4), 245-255. https://doi.org/10.1016/S0897-1897(03)00054-5

\section{Copyrights}

Copyright for this article is retained by the author(s), with first publication rights granted to the journal.

This is an open-access article distributed under the terms and conditions of the Creative Commons Attribution license (http://creativecommons.org/licenses/by/4.0/). 